\title{
Near continuum flows over a sphere
}

\author{
Chunpei Cai ${ }^{\mathrm{a}, *}$, Quanhua Sun ${ }^{\mathrm{b}}$ \\ ${ }^{a}$ Department of Mechanical \& Aerospace Engineering, Jett Hall 110, MSC 3450, New Mexico State University, Las Cruces, NM 88003, United States \\ ${ }^{\mathrm{b}}$ State Key Laboratory of High Temperature Gas Dynamics, No. 15 Beisihuanxi Road, Institute of Mechanics, Chinese Academy of Sciences, Beijing 100190, China
}

\section{A R T I C L E I N F O}

\section{Article history:}

Received 28 June 2014

Received in revised form 5 November 2014

Accepted 4 January 2015

Available online 29 January 2015

\section{Keywords:}

Flow computation

Navier-Stokes equations

Slip flows

\begin{abstract}
A B S T R A C T
In this paper, the problem of near continuum gas flows over a sphere is investigated numerically. Three types of boundary conditions for the sphere surface are adopted: (1) non-slip and constant temperature surface; (2) velocity slip with considerations of velocity gradient, and temperature jump at the surface; and (3) velocity slip with considerations of both velocity and temperature gradients, as well as temperature jump at the surface. Navier-Stokes equations in a cylindrical coordinate system for compressible flows are adopted with the Roe numerical scheme. The numerical simulation results include coefficient distributions for surface pressure, friction, heat flux, velocity slip, temperature jump and total drag. The results are obtained with different free stream Knudsen and Mach numbers. Several conclusions include: (i) the third surface boundary condition does not create significant differences from the second type, (ii) however, an adoption of non-slip or a slip surface boundary condition can create significant differences in $C_{f}, C_{q}$ and $C_{D}$.
\end{abstract}

(c) 2015 Elsevier Ltd. All rights reserved.

\section{Introduction}

Gas flow over a sphere is a fundamental fluid dynamic problem with numerous applications. Examples include, but are not limited to, (i) droplets or particulate matters in a smog; (ii) liquid droplets during a spray; (iii) aluminium powders in combustion chambers; (iv) solar winds passing a moon of no gravity; (v) mixing processes in chemical engineering; and (vi) heavy materials separation in nuclear engineering. Many of these examples are related to rarefication effects, which can be expressed with the Knudsen (Kn) number [1]:

$K n=\lambda / L$,

where $\lambda$ is the mean free path, for the hard sphere model $\lambda=1 /\left(\sqrt{2} \pi d^{2} n\right), L$ a characteristic length, $d$ the molecular diameter, and $n$ the number density. According to different $K n$ numbers, flows can be classified as: (i) continuum flow $(K n<0.001)$ which can be solved by the Navier-Stokes Equations (NSEs) and the conditions of no-velocity-slip, no-temperature-jump boundary conditions at the surface; (ii) slip flow $(0.001<K n<0.01)$, where the NSEs are still applicable but the boundary conditions at the surface shall be modified by considering some gradient effects, examples include flows related with Micro-Electro-Mechanical Systems (MEMS); (iii) for most transitional flows $(0.01<K n<10)$, numerical simula-

\footnotetext{
* Corresponding author.

E-mail address: ccai@nmsu.edu (C. Cai).
}

tions shall be used for investigations; and (iv) free molecular flows $(K n>10)$, where both numerical and analytical results are applicable. The current status for development of low speed sphere flows can be summarized as follows: (i) in the continuum flow regime, there are many successful investigations, numerically, theoretically, and experimentally; (ii) in the slip flow regime, there are some recent developments for numerical and experimental investigations which provide some new insights; (iii) in the transitional flow regime, almost all studies are numerical simulations; for example, with the direct simulation Monte Carlo (DSMC) [1] method; and (iv) free molecular flow, analytical expressions for surface properties [2] for a diffusive or specular reflective sphere were obtained about half a century ago. The flowfield properties (i.e., density, velocity, pressure and temperature), were investigated with the gaskinetic methods, and some such analytical methods are documented [3]. However, only recently [4], have the detailed flowfield properties for flows over a diffusive been reflective sphere were evaluated and validated with several DSMC simulations. In the literature, there are some efforts to develop unified solvers (e.g. $[5,6])$, aiming to simulate the full Kn number flows, and several kinds of gaskinetic schemes for near continuum flows, e.g. [7].

In this paper, the problem of slightly rarefied gas flows over a sphere will be investigated numerically. In the literature, theoretical studies are rare, and there are scarce numerical studies with different simplifications. One major focus of this paper is about analytical treatment of the velocity-slip and temperature-jump boundary conditions. In history, more than one hundred years 
ago, Maxwell [8] deduced the well-known velocity slip boundary condition for macroscopic conservation variables, with an extrapolated asymptotic incident distribution function, after which many progresses have been made in the related fields, such as the high-order slip conditions [9]. Further, the Maxwell-Smoluchowski temperature jump boundary condition (with full accommodation) can also be recovered automatically [10]. Later, we will address the differences between this work and those in the literature.

In section II, a detailed problem statement, governing equations, boundary conditions and numerical schemes will be provided and discussed; in section III, many comparisons and discussions on the results will be performed; and in the last section, summaries and conclusions from the work will be presented.

\section{Problem descriptions and the simulation scheme}

The problem is illustrated as Fig. 1: slightly rarefied, or near continuum, gas flows over a sphere of a radius $R_{1}$. The flowfield is symmetric; hence, only the upper flowfield is studied. There are three boundaries: (i) free stream flows entering the outer circular boundary with a radius of $R_{2}$, with given flowfield properties, e.g., $\rho_{\infty}, U_{\infty}, T_{\infty}$, and $p_{\infty}$; (ii) symmetric center lines; and (iii) sphere surface with boundary conditions of nonslip/slip velocity and constant/jump temperature.

To be general, compressibility with different free stream Mach numbers will be considered, and the governing equation for mass flux is included in this study. We do not adopt an incompressible fluid simulation solver because by default it assumes the density is constant; however, rarefied gas flows include density variations. For the simulations, the outer external boundary edge is set to be very large compared with the sphere diameter, and setting the free stream properties at the outer edge is sufficient. A full set of NSEs are adopted to solve the coupling flow and temperature fields simultaneously with density, flowfield velocity, and temperature, $\rho, U, V$, and $T$. The flow is essentially axisymmetric, and the governing equations are:

$\frac{\partial U}{\partial t}+\frac{\partial\left(E_{i n}-E_{v}\right)}{\partial x}+\frac{\partial\left(F_{i n}-F_{v}\right)}{\partial r}=\frac{G}{r}$,

$U=\left(\begin{array}{c}\rho \\ \rho u \\ \rho v \\ E\end{array}\right), E_{i n}=\left(\begin{array}{c}\rho u \\ \rho u^{2}+p \\ \rho u v \\ u(E+p)\end{array}\right), F_{i n}=\left(\begin{array}{c}\rho v \\ \rho u v \\ \rho v^{2}+p \\ v(E+p)\end{array}\right)$,

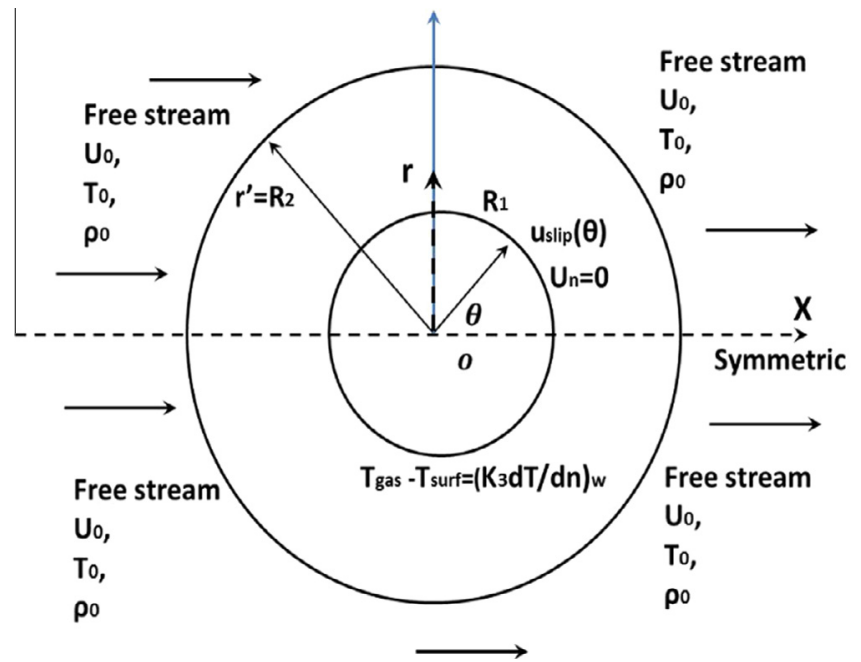

Fig. 1. Model schematic: sphere (cut through) and boundary conditions.

$$
\begin{aligned}
& E_{v}=\left(\begin{array}{c}
0 \\
\tau_{x x} \\
\tau_{x r} \\
u \tau_{x x}+v \tau_{x r}+q_{x}
\end{array}\right), F_{v}=\left(\begin{array}{c}
0 \\
\tau_{x r} \\
\tau_{r r} \\
u \tau_{x r}+v \tau_{r r}+q_{r}
\end{array}\right) \text {, } \\
& G=\left(\begin{array}{c}
-\rho v \\
\tau_{x r}-\rho u v \\
\tau_{r r}-\tau_{r x}-\rho v^{2} \\
u \tau_{x r}+v \tau_{r r}+q_{r}-v(E+p)
\end{array}\right) \text {, } \\
& \tau_{x x}=-\frac{2}{3} \mu\left(2 \frac{\partial u}{\partial x}-\frac{\partial v}{\partial r}-\frac{v}{r}\right), \quad \tau_{r r}=-\frac{2}{3} \mu\left(2 \frac{\partial v}{\partial r}-\frac{\partial u}{\partial x}-\frac{v}{r}\right), \quad \tau_{x r}=\mu\left(\frac{\partial u}{\partial r}+\frac{\partial v}{\partial x}\right), \\
& \tau_{r x}=-\frac{2}{3} \mu\left(\frac{\partial u}{\partial x}+\frac{\partial v}{\partial r}+\frac{v}{r}\right), \quad q_{x}=k \frac{\partial T}{\partial x}, \quad q_{r}=k \frac{\partial T}{\partial r},
\end{aligned}
$$

where $E$ is internal energy. The above formulas are similar to those for two-dimensional flows; the differences include several extra terms within the shear stress tensor and extra source terms.

The variable hard sphere (VHS) model [1] is used to model the viscosity variations with temperature, and the thermal conductivity coefficient $k$ changes correspondingly:

$\mu(T)=\mu_{r e f}\left(T / T_{\text {ref }}\right)^{\omega_{1}}, k(T)=\frac{\gamma R}{\gamma-1} \frac{\mu}{\operatorname{Pr}}$,

where $\mu_{\text {ref }}$ is the reference viscosity at a specific temperature $T_{\text {ref }}, \omega_{1}$ is the viscosity index, and Pr the Prandtl number. The equation of state is adopted to link the pressure, temperature and density.

The boundary conditions for the problem are listed as:

$T=T_{\infty} ; p=p_{\infty} ; \quad U=U_{\infty}, \rho=\rho_{\infty} ;$ at farfield,

$v=0 ; \quad \frac{\partial u}{\partial r}=\frac{\partial P}{\partial r}=0 ; \frac{\partial T}{\partial r}=0, \quad$ symmetric lines at the bottom.

There are some different surface boundary conditions. For convenience, the direction normal to the sphere surface is denoted as " $n$ "; "s" is adopted to represent the distance along the sphere surface, by starting from the front stagnation point, and " $\theta$ " is used to represent the corresponding polar angle, starting from the stagnation point, along a clockwise direction:

$u_{n}=0, \quad u_{\theta}=0, \quad T_{g}=T_{w}, \quad$ non-slip and constant temperature,

$u_{n}=0 ; \quad u_{\theta}=K_{1} \frac{\partial u_{\theta}}{\partial n}+\frac{K_{2}}{T_{g}} \frac{\partial T_{g}}{\partial s}$,

$T_{g}-T_{w}=K_{3} \frac{\partial T_{g}}{\partial n}$, velocity slip at sphere surface,

where $R_{1}$ is the sphere radius, and

$K_{1}=\frac{2-\sigma_{M}}{\sigma_{M}} \lambda ; K_{2}=\frac{3}{4} v ; K_{3}=\frac{2-\sigma_{T}}{\sigma_{T}} \frac{2 \gamma}{\gamma+1} \frac{\lambda}{\operatorname{Pr}}$.

In the past, in the velocity slip and temperature jump boundary formulas, Eq. (9), the term with the temperature gradient, $\partial T / \partial s$, was considered to be smaller than the velocity gradient, $\partial u_{\theta} / \partial n$; hence, it is usually set to zero for simplicity. In this work, such a treatment is denoted as a "partial slip" surface model. A more comprehensive model with both velocity and temperature gradients is also investigated as a "fully slip" surface model. The latter preserves the temperature gradient $\partial T / \partial s$, because around the front stagnation point, the velocity is zero, while the temperature is the highest; hence, some temperature variations along the front side of the sphere surface are expected, and may contribute to the slip velocity. One major goal of this paper is to compare the contribution due to the latter two different surface boundary conditions. The numerical simulation scheme in this work was the Roe's approximate Riemann solver [11,12]. The flow field is divided 


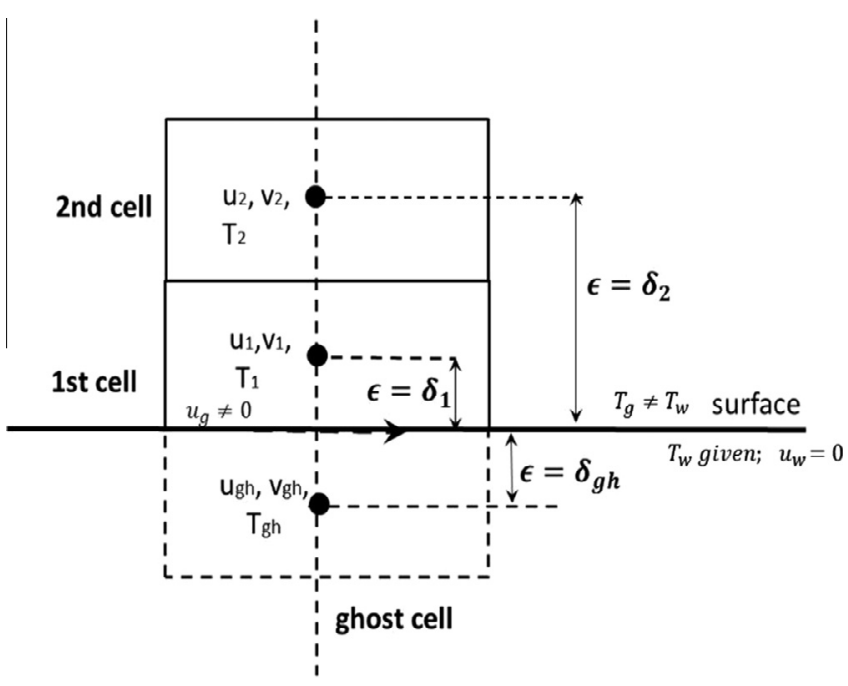

Fig. 2. Illustration for near surface ghost cell treatment.

into small cells, and the finite volume method is adopted for the simulation. Ghost cells are distributed along the simulation domain boundaries, and the one at the wall surface is illustrated as Fig. 2. At the surface, the gas velocity $u_{g}$ and temperature $T_{g}$ are not the same as the corresponding surface properties, and both will be updated at each numerical simulation step.

The treatment of the wall boundary conditions in this work is crucial. For the non-slip boundary condition, the velocity and temperature gradients are important properties, and they are handled with second order extrapolations. At locations sufficiently close to the surface, the velocity is nonslip and can be assumed to behave as:

$u(\epsilon)=a+b \epsilon+c \epsilon^{2} ; u(\epsilon=0)=0, u\left(\epsilon=\delta_{1}\right)=u_{1}, u\left(\epsilon=\delta_{2}\right)=u_{2}$,

where $\epsilon$ is the distance from the sphere surface along the normal direction; and $\delta_{1}, \delta_{2}$ are the distances from the surface to the centers of the 1st and 2nd layers of cells (see Fig. 2), with two computed properties $u_{1}\left(\epsilon=\delta_{1}\right), u_{2}\left(\epsilon=\delta_{2}\right)$ at the two cell centers. The coefficients of $a, b$, and $c$ can be derived. The ghost cell properties are updated to satisfy the nonslip and constant temperature boundary conditions:

$u_{g h}\left(\epsilon=-\delta_{1}\right)=-u\left(\epsilon=\delta_{1}\right), v_{g h}\left(\epsilon=-\delta_{1}\right)=-v\left(\epsilon=\delta_{1}\right)$,

$T_{g h}\left(\epsilon=-\delta_{1}\right)=2 T_{w}-T\left(\epsilon=\delta_{1}\right)$.

For the velocity slip and temperature jump boundary conditions, the temperature solution shall be solved first as its gradient presents in the velocity slip boundary conditions. It can be assumed that at locations sufficiently close to the sphere surface, the relation between the temperature and its gradient is also generally applicable; hence, the 3rd formula in Eq. (9) can be treated as a first order ordinary differential equation (along the normal ndirection) for the temperature, and the analytical solution for temperature is:

$T_{g}(\epsilon)=\left(T_{g}\left(\delta_{1}\right)-T_{w}\right) e^{\left(\epsilon-\delta_{1}\right) / K_{3}}+T_{w}$,

where $T_{g}\left(\delta_{1}\right)$ is the corresponding gas bulk temperature at the center for the first layer cells. Then, it is evident that:

$\left.\frac{d T_{g}}{d n}\right|_{\epsilon=0}=\frac{e^{-\delta_{1} / K_{3}}}{K_{3}}\left(T_{g}\left(\delta_{1}\right)-T_{w}\right)$,

or may be obtained from Eq. (9) quite conveniently. As the results from Eq. (13), the gas temperature distributions, $T_{g}(\epsilon=0)$, along the surface are obtained; hence, $\partial T / \partial s$ can be computed with a simple finite difference method.

For the "partial" velocity slip model, the treatment for the velocity at the sphere surface is quite similar with the temperature distributions. The 2nd formula in Eq. (9) can be considered as a 1st order ordinary differential equation as well, and the solution is:

$u_{\theta}(\epsilon)=u_{\theta}\left(\delta_{1}\right) e^{\left(\epsilon-\delta_{1}\right) / K_{1}}$,

correspondingly, for the "full" slip model which further considers the temperature gradient, the solution is:

$u_{\theta}(\epsilon)=u_{\theta}\left(\delta_{1}\right) e^{\left(\epsilon-\delta_{1}\right) / K_{1}}+\left.\left(\frac{K_{2}}{T_{g}} \frac{\partial T_{g}}{\partial s}\right)\right|_{\epsilon=0}$.

The above semi-analytical formulas only require some input from the 1 st and the 2 nd cells adjacent to the surface. Within each numerical simulation step, they are updated; hence, the computations are quite convenient. Temperature, velocity, and their gradients at the surface are via interrelated and the boundary effects to the flowfield via the flux contributions from the ghost cells.

We adopted Roe's approximate Riemann solver [11,12] for the simulations. The flow field is divided into small cells, and the finite volume method is adopted for the simulation. Ghost cells are distributed at the three boundaries; and one at the wall surface is illustrated as Fig. 2. At the surface, the gas velocity $u_{g}$ and temperature $T_{g}$ are not the same as the corresponding surface properties, and are updated within every simulation step.

Eqs. (2)-(4) can be expressed with the following discrete format:

$\frac{\partial U_{j}}{\partial t}=S_{j}-\frac{1}{A}\left[\sum_{l=1}^{4}\left(E_{i n} \delta r-F_{\text {in }} \delta x\right)_{l}\right]_{j} \equiv \operatorname{Res}_{j}$,

where $S_{j}$ contains $G_{j} / r, E_{v}, F_{v}$; the very right hand side term is defined as "Residual" for the $j_{\text {th }}$ cell, $A$ is the cell area.

\section{Results and discussions}

Several simulations were performed, with those three types of surface boundary conditions. During the simulations, $R_{2}$ is set to $20 R_{1}$, the Mach number is set to small values to achieve an incompressible flow state, and the Kn number is set between 0.001 and 0.01 , within the velocity slip and temperature jump regime. The

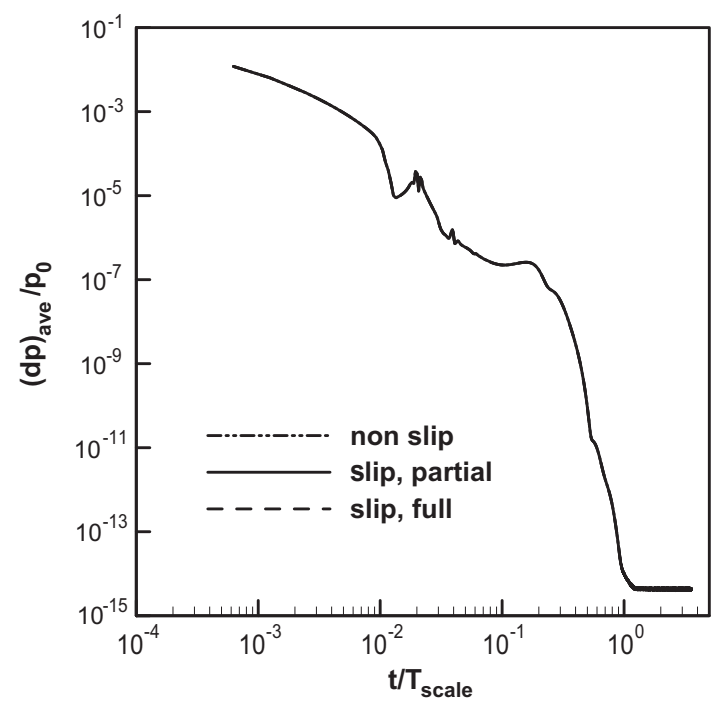

Fig. 3. Numerical simulation residual development histories: with $K n=0.001$ and $M a=0.05$. 
simulation results obtained with nonslip boundary conditions are for comparison, while the simulation results using the slip boundary condition are more reliable. Both mesh resolution and size dependence are studied. A $200 \times 200$ mesh size along the circumferential and radial directions is adopted to discrete the flowfield, and mesh convergency examinations indicate such a mesh size is sufficient to resolve the flowfield.

Fig. 3 shows the residual development histories during one set of numerical simulations with the three kinds of velocity boundary conditions, with $K n=0.001$ and $M a=0.05$. There are minor differences among the three curves; however, they are not appreciable in log-scales. The residuals decrease almost twelve orders smaller and the convergence is evident. The $X$-axis is normalized by the characteristic time, while the $y$-axis by the free stream pressure:

$t^{*}=t /\left(R_{1} / U_{\infty}\right), p^{*}=\frac{1}{p_{\infty}} \sqrt{\frac{1}{N_{\text {cell }}} \sum_{i=1}^{N_{\text {cell }}}(\Delta p)_{i}^{2}}$.

Fig. 4 shows the normalized pressure distributions with the full velocity slip boundary condition model with $K n=0.001$ and $M a=0.05$. The stagnation pressure is slightly larger than other locations, and the smallest pressure adjacent to upper right surface indicates larger flow velocity happening there. Fig. 5 shows the Mach number contours with the model of full velocity slip boundary condition. In front of the sphere, flows are compressed or squeezed, due to the narrower passage between streamlines; hence, the streamline gradient along the sphere normal direction varies. The streamlines are not only functions of $\theta$, but also of $r$ and other variables. At the wake zone with vortex circulations, the average Mach number decreases. Evidently, as shown in this figure, flow separations occur on the surface and a small vortex is plotted. Fig. 6 shows the normalized v velocity component, with the full slip boundary condition. These contours change from positive to negative values and are effective to show flowfield patterns.

The above three figures are for the combinations of $K n=0.001$ and $M a=0.05$ with the full velocity slip boundary conditions, while the next five figures present various surface properties by comparing the partial and full slip boundary conditions. Fig. 7 shows sphere surface pressure distributions, $C_{p}(\theta)=\left(p(\theta)-p_{\infty}\right) /$ $\left(\rho_{\infty} U_{\infty}^{2} / 2\right)$, for the non slip, partial and full slip boundary conditions. Essentially there are no appreciable differences. Fig. 8 shows

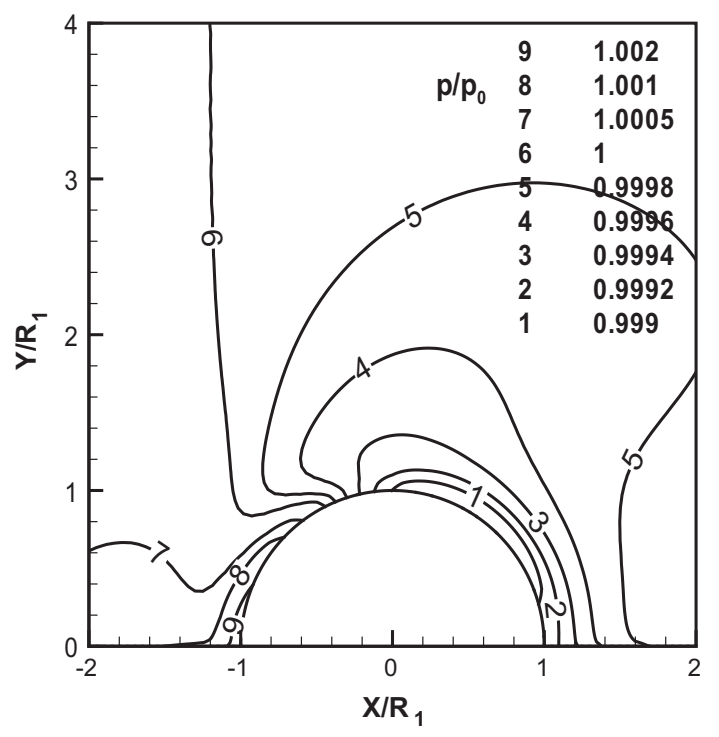

Fig. 4. Normalized pressure distributions, $p / p_{0}$, full velocity slip condition, with $K n=0.001$ and $M a=0.05$

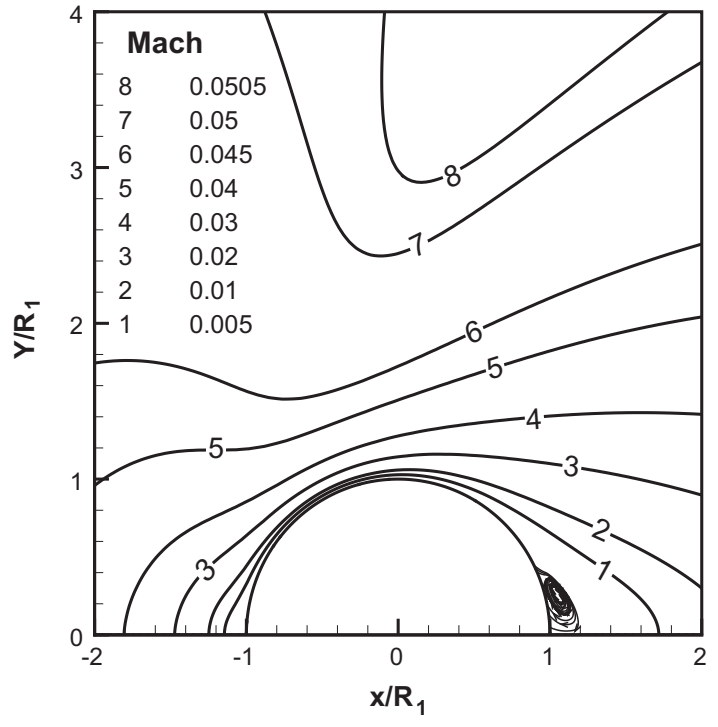

Fig. 5. Mach number contours and an attached vertex at the back of the sphere, full slip condition, with free stream conditions $K n=0.001$ and $M a=0.05$.

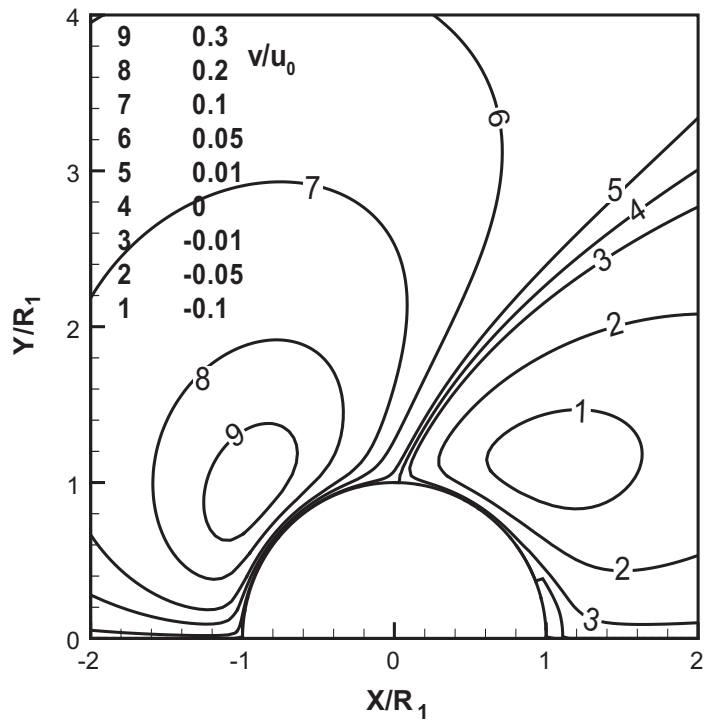

Fig. 6. Normalized velocity component $v / U_{0}$, full slip condition, with $K n=0.001$ and $M a=0.05$.

the normalized surface friction distributions, $C_{f}(\theta)=\tau(\theta)$ / $\left(\rho_{\infty} U_{\infty}^{2} / 2\right)$. The friction coefficient for the non slip boundary condition is significantly larger than the slip boundary condition results. A larger shear stress coefficient at the front side indicates larger velocity changes in the local flowfield; and $C_{f}=0$ around $150^{\circ}$ reflects a flow separation there. Fig. 9 shows the sphere surface heat transfer coefficient $C_{q}(\theta)=k(\partial T / \partial n) /\left(\rho_{\infty} U_{\infty}^{3} / 2\right)$. The heat flux for the non-slip boundary case has a much larger value than the slip boundary condition results; this may be due to the larger friction. Eq. (9) indicates that for both the partial and full flip scenarios, the heat flux is proportional to the surface temperature gradient and the surface temperature differences. The stagnation temperature is the highest, indicating the highest stagnation point heat flux. Fig. 10 shows normalized sphere surface slip velocities, $u_{g}(0) / u_{\infty}$, and the differences between the partial-slip and full-slip conditions are minor. Along the surface, around $\theta \approx 150^{\circ}$, the slip velocity value changes signs, reflecting a flow separation. Fig. 11 


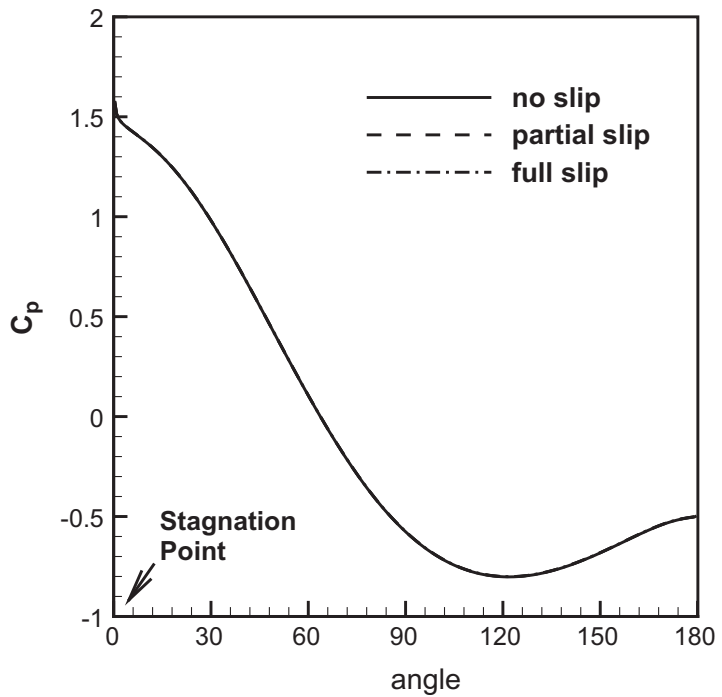

Fig. 7. $C_{p}$ with non-slip, "partial" and "full" slip boundary conditions, respectively, at $K n=0.001$ and $M a=0.05$.

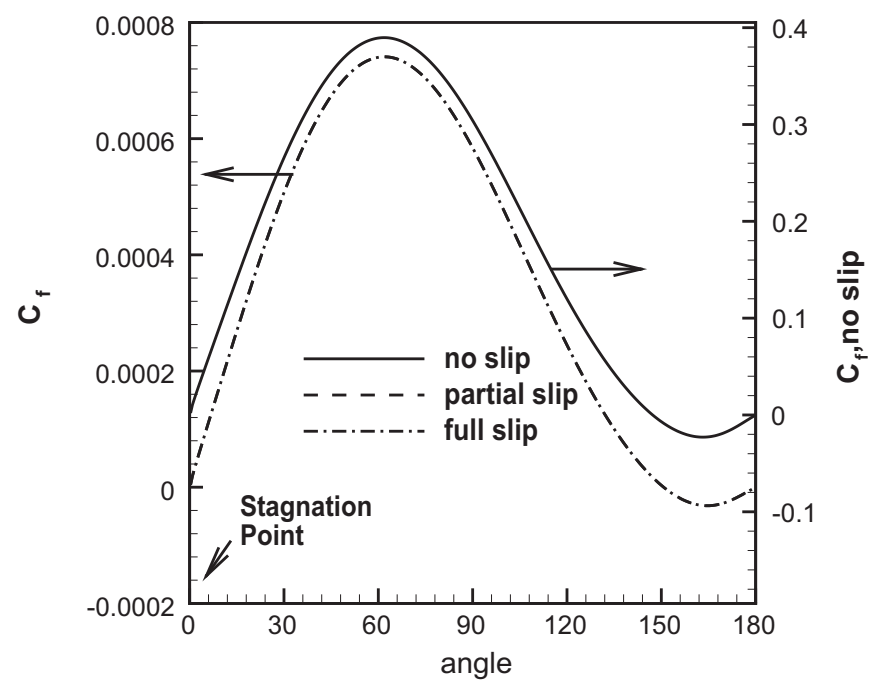

Fig. 8. $C_{f}$, with non-slip, "partial" and "full" slip boundary conditions, respectively, at $K n=0.001$ and $M a=0.05$.

shows the normalized temperature jump along the sphere surface, $\left(T_{g}(\epsilon=0)-T_{w}\right) / T_{w}$. Eq. (9) shows that the temperature is actually proportional to the local gradients, and the full and partial slip velocity boundary conditions yield almost the same patterns.

The above five figures illustrate that the full and partial velocity slip conditions create almost identical flowfield and surface property patterns, only small differences happen in the slip velocity. This indicates that the term containing the temperature gradient $\partial T / \partial s$, in Eq. (9), provides negligible contribution to the flow solutions. However, as shown, slip and non-slip boundary conditions truly create different results of larger friction, total drag, and heat flux at the sphere surface.

One major concern about flows over a spherical object is the total drag coefficient. For the total drag coefficient variations with the free stream Mach and the Kn numbers, the "partial" and "full" velocity slip boundary conditions do not create differences, and this fact can be clearly explained with Figs. 7 and 8. For compressible flows in the continuum regime, there are some curve fitting results between the Reynolds number and the drag coefficient [15]:

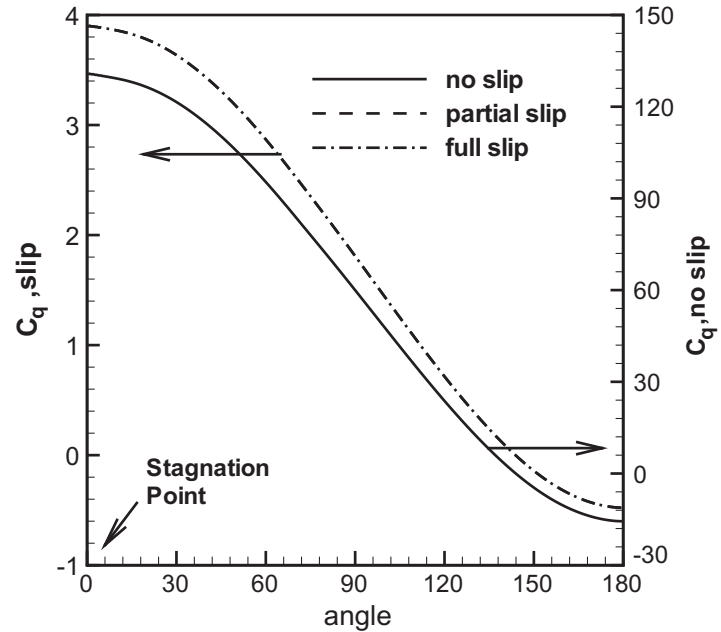

Fig. 9. $C_{q}$ distributions with non-slip, "partial" and "full" slip boundary conditions, respectively, at $K n=0.001$ and $M a=0.05$.

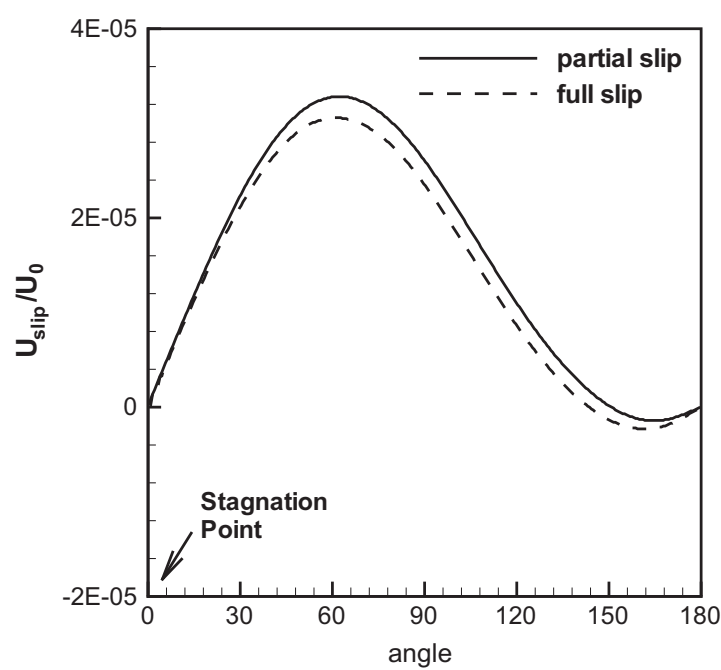

Fig. 10. Normalized $u_{\text {slip, }}$ with "partial" and "full" slip boundary conditions, respectively, at $K n=0.001$ and $M a=0.05$.

$C_{D} \approx \frac{24}{R e}+\frac{6}{1+\sqrt{R e}}+0.4 ; 0<R e<2 \times 10^{5}$.

In addition, there is a relation among the $K n, M a$ and $R e$ numbers for compressible flows:

$R e=\frac{\rho_{0} U_{0} D_{0}}{\mu_{0}}=\sqrt{\frac{\pi \gamma}{2}} \frac{M a}{K n}$.

It is very similar to the Von Karman relation [16,17].

It is heuristic to understand the patterns with these parameters, $\mathrm{Kn}, \mathrm{Ma}$, and Re; for example, an increase of the Mach number leads to a smaller total drag coefficient. Fig. 12 shows the drag coefficients $C_{D}$ with different Reynolds number, which can be computed from $M a$ and $K n$ numbers via Eq. (20). The figure illustrates several features:

1. using non-slip and slip boundary conditions can lead to quite appreciable differences, even at the $K n=0.001$ limiting value;

2. at large Re number, e.g. $R e>150$, the simulation results with and without velocity slip boundary conditions agree relatively better with White's curve fitting results [15], i.e., Eq. (19); -this 


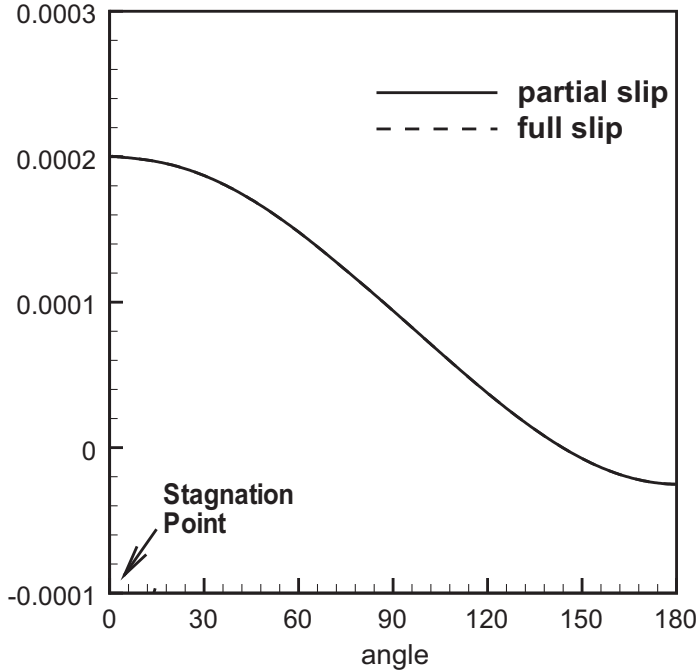

Fig. 11. Temperature jump at the sphere surface, with "partial" or "full" velocity slip surface conditions, respectively, at $K n=0.001$ and $M a=0.05$.

indicates the simulation algorithm including the sphere surface condition treatment, are reliable and the simulation results are solid;

3. the Re number range is limited by Eq. (20), the largest Re value happens with the largest Mach number $=0.3$, and the smallest Kn number $=0.001$, to satisfy the incompressible and near continuum flow state conditions. Beyond this largest Re number, the flows can be either compressible or not near continuum. Close to this limit, the curve fitting $C_{D}$ results vary mildly, and the agreement with numerical simulations is relatively good;

4. with a smaller Ma number or larger Kn number, the flow regime has smaller Re number; unfortunately, the curve-fitting formula by White [15] changes rapidly with small Re numbers, and the simulation data scatter around the curve. This indicates at smaller Re number range, a consideration of rarefication effects may lead to much larger differences;

5. the drag coefficients distribute within a wide range due to the Kn number effect. With a fixed Reynolds number, rarefication can create appreciable effect on drag coefficients. Consequently, cautions shall be exercised during the simulations, especially for the selection of velocity slip or non-slip surface boundary conditions.

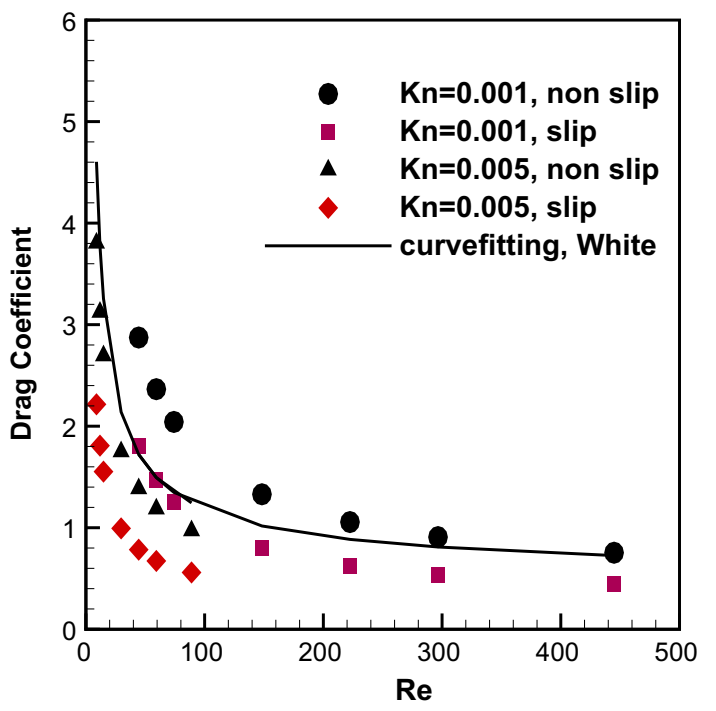

Fig. 12. Drag coefficient variations with different Reynolds numbers.
Table 1

Surface flow separation angles, varying with different Kn and Ma numbers, in degree $\left({ }^{\circ}\right)$.

\begin{tabular}{llllllll}
\hline \multirow{2}{*}{ Kn } & Ma & & & & & & \\
\cline { 2 - 7 } & 0.3 & 0.2 & 0.15 & 0.1 & 0.05 & 0.04 & 0.03 \\
\hline 0.001 (non slip) & 117.3 & 124.6 & 129.3 & 135.9 & 147.0 & 151.0 & 156.3 \\
0.001 (partial slip) & 122.1 & 128.3 & 132.9 & 138.3 & 150.1 & 154.8 & 160.5 \\
0.001 (full slip) & 122.1 & 128.3 & 132.9 & 138.9 & 150.1 & 154.2 & 161.0 \\
0.005 (non slip) & 124.5 & 131.1 & 135.3 & 141.3 & 153.8 & 159.3 & No separation \\
0.005 (partial slip) & 128.1 & 133.5 & 138.4 & 144.9 & 158.1 & 165.3 & No separation \\
0.005 (full slip) & 128.1 & 133.5 & 138.3 & 144.9 & 158.0 & 165.3 & No separation \\
\hline
\end{tabular}

Table 1 shows the flow separation angles with different Kn and Mach numbers, by using the sphere diameter as the characteristic length. The angles are measured along the surface, clockwise-ly, starting from the stagnation point. It is evident that: (i) With a fixed Kn number, an increase of the Mach number results in an earlier separation; this may be related to the larger Re numbers; (ii) for a fixed Mach number, the non-slip boundary condition create earlier separations; (iii) a larger Kn number delays separations due to a smaller Re number; (iv) there may be no separations at all with high Kn but low Mach numbers, i.e. with a smaller Reynolds number; and (v) the separation angles for partial and full slip boundary conditions are essentially the same. In addition, the flow simulations are based on laminar flow, and there may be flow instabilities which are not considered; hence, the separation angles are only for reference.

Before the end of this section, we would like to point out the differences between this work and the past ones. In this work, the velocity slip and temperature jump boundary conditions are treated as ordinary differential equations, at the neighborhood of the surface; also we concentrate on special case of a classical problem of low Reynolds number sphere flow. In the work by $\mathrm{Li}$ and $\mathrm{Fu}[5,6]$, the surface boundary conditions are treated with the gaskinetic theory, the results are related to the work here; however, they relied on numerical simulations, and used high Reynolds number flows over a sphere as a test case. There are experimental results available in the literature to validate their results, but the drag coefficients are different. There is other different treatment on the velocity slip boundary conditions. For example, Myong [13] argued to achieve true Maxwellian boundary conditions, higher order terms which may account for the curvature effect shall be added. In our modeling, we consider that effect may already be considered in the governing Eqs. (4) and (5), i.e., the source terms and the extra shear stress terms. In this sense, the work in paper maybe not exactly base on a Maxwellian boundary condition, however, the velocity boundary conditions are much concise, and its simplicity allows the treatment as solutions to an ODE at the neighborhood of the surface possible. Another paper [14] argued higher order but more complex terms can be added into the slip boundary conditions, such as second order velocity gradients and density gradient, however, the work is very complex and the effects seem not appreciable. It can be considered as a good review paper for higher order velocity-slip boundary conditions. For the work by others, e.g. Maxwell [8], Kennard [10], and Beskok [9], are rather pure theoretical or modeling, without real validations.

\section{Summary}

In this paper, we presented investigations on compressible, near continuum gas flows over a sphere. Three types of surface boundary conditions, non-slip, partial or full slips, were incorporated with the full set of Navier-Stokes equations. The chosen simulation parameters are within special $\mathrm{Kn}$ and Ma number ranges, to create 
near continuum and incompressible flows. The numerical scheme was the Roe's approximate solver. The adoptions of surface boundary conditions created several analytical solutions for the temperature and velocity at locations close to the sphere surface.

In this study, we investigated the flow and temperature fields without adopting many assumptions and simplifications which were used by many other papers in the literature. The results indicated that the full or partial slip boundary conditions lead to negligible effects on the flowfield and surface properties; hence, we confirm that the traditional treatment of slip boundary conditions is proper. However, adoptions of velocity slip and non-slip boundary conditions may lead to quite differences in $C_{f}, C_{q}$, and $C_{D}$, -we shall be quite cautious during simulations of near continuum flows over a sphere.

\section{References}

[1] Bird GA. Molecular gasdynamics and numerical simulation methods. Oxford Universtiy Press; 1994.

[2] Schaaf SA, Chamber PL. Flow of rarefied gases. Princeton University Press; 1961.

[3] Cercignani C. Rarefied gas dynamics: from basic concepts to actual calculations. Cambridge University Press; 2000
[4] Cai C, Khasawneh KR, Liu H, Wei M. Collisionless gas flows over a cylindrical or a spherical object. J Spacecraft Rockets 2009;46(6).

[5] Li ZH, Zhang HX. Study on gas kinetic unified algorithm for flows from rarefied transition to continuum. J Comput Phys 2004;193:708-38.

[6] Li ZH, Zhang HX. Gas numerical studies of three-dimensional complex flows on a spacecraft re-entry. J Comput Phys 2009;228:1116-38.

[7] Li QB, Fu S. Application of implicit BGK scheme in near continuum flows. Int Comput Fluid Dynam 2006;20(6):453-61.

[8] Maxwell JC. On stress in rarefied gases arising from inequalities of temperature. Philos Trans Roy Soc Lond 1979;170:231-56.

[9] Kardiadakis G, Beskok A. Micro flows: fundamental and simulations. New York: Springer-verlag: 2002.

[10] Kennard EH. Kinetic theory of gases: with an introduction to statistical methods. New York: McGraw-Hill; 1938.

[11] Roe PL. Approximate Riemann solvers, parameter vectors, and different schemes. J Comput Phys 1981;43:357-72.

[12] Hirsch C. Numerical computation of internal and external flows, 8th ed., vol. 2. Wiley \& Sons; 2002.

[13] Myong RS. Gaseous slip models based on the Langmuir adsorption isotherm. Phys Fluids 2004;16(1):104.

[14] Lockerby D, Resse J, Emerson D, Barber R. The velocity boundary condition at solid walls in rarefied gas calculations. Phys Rev E: Stat Phys Plasma Fluids Relat Interdisc Top 70(1): 017403, ISSN: 1063-651X

[15] White F. Viscous fluid flow. 2nd ed. 1992: McGraw Hill; 1992.

[16] Tsien HS. Similarity laws of hypersonic flows. J Math Phys 1946;25:247-52.

[17] vonKarman T. From low-speed aerodynamics to astronautics. Pergamon Press; 1963. 